\title{
Theory of electric-field-induced metal-insulator transition in doped manganites
}

\author{
R. Y. Gu, ${ }^{1}$ Z. D. Wang, ${ }^{1,2}$ and C. S. Ting ${ }^{1}$ \\ ${ }^{1}$ Texas Center for Superconductivity and Department of Physics, University of Houston, Houston, Texas 77204 \\ ${ }^{2}$ Department of Physics, The University of Hong Kong, Hong Kong, China
}

(Received 24 September 2002; published 1 April 2003)

\begin{abstract}
The insulator to metal transition (IMT) induced by the application of an electric field in doped manganites is investigated theoretically. Starting from the double-exchange mechanism with the long-range Coulomb interaction included, we find that the electric field may suppress the charge ordering and drive the system from the antiferromagnetic and charge-ordered state with an energy gap at the Fermi level to the ferromagnetic and gapless state, resulting in the IMT. A numerical simulation is performed for manganite films with intrinsic inhomogeneities, and an important impact of the inhomogeneities on this electric-field-induced transition is obtained. Our results can naturally account for the recently observed electric-filed-induced IMT phenomenon in manganites.
\end{abstract}

DOI: 10.1103/PhysRevB.67.153101

The carrier-doped manganites with perovskite structure, $R_{1-x} A_{x} \mathrm{MnO}_{3}$ (where $R$ and $A$ are rare and alkaline-earth ions, respectively) have attracted much attention maily due to fruitful physical properties that they exhibit. One particular and interesting property is the insulator to metal transition (IMT) observed in some narrow bandwidth manganites, which can be triggered by the variation of the doping, ${ }^{1}$ temperature, ${ }^{2}$ and the application of some external forces, such as a magnetic field, ${ }^{3}$ a higher pressure, ${ }^{4}$ an exposure to $x$ rays ${ }^{5}$ or visible light, ${ }^{6}$ and even an electric field. ${ }^{7-12}$ In the presence of magnetic field, the observed "colossal magnetoresistance" phenomenon can be understood qualitatively in terms of the double-exchange (DE) mechanism: the strong on-site Hund's coupling aligns the spin of the extra $e_{g}$ electron on each $\mathrm{Mn}^{3+}$ parallel to the corelike $t_{2 g}$ spins so that the transfer of this electron to the neighboring $\mathrm{Mn}^{4+}$ site depends on the relative configuration of the two neighboring core spins, which can be affected by the magnetic field. Although the DE model provides a simple but natural explanation for the IMT induced by the magnetic field (or by the pressure) via enhancing the effective electron hopping, whether it can also account for the IMT induced by an electric field (or light) is not so clear as there is no direct connection between the latter and the magnetic configuration of the system. Furthermore, unlike the magnetic field and high pressure which cause the whole sample to undergo the phase transition, it was reported experimentally that the application of the electric field results in an IMT, where a nucleation of metallic patches that form one or more filamentary metallic paths may occur. ${ }^{6,7}$ According to Ref. 7 the ground state of $\mathrm{Pr}_{1-x} \mathrm{Ca}_{x} \mathrm{MnO}_{3}$ with $x=0.3-0.5$ is a charge-ordered antiferromagnetic $(\mathrm{AF})$ insulator with the charge ordering $(\mathrm{CO})$ having the pattern similar to that at half doping $(x=0.5)$. The application of a static electric field triggers the collapse of the low temperature, electrically insulating $\mathrm{CO}$ state to a metallic ferromagnetic (FM) state. After being switched into the much lower-resistance state from the higher-resistance state at a threshold voltage $V_{t h}$ in the increasing-voltage scan, the resistance shows hysteretic behavior and then returns to the original value before switching in the decreasingvoltage scan. This kind of switching effect, which (or a simi-
PACS number(s): 71.30.+h, 71.10.-w, 75.10.-b

lar one) was also observed by others, ${ }^{8-12}$ is very interesting and may have potential applications in micro-electronics or nanoelectronics including high-density nonvolatile memory.

So far, although many theoretically efforts have been devoted to the understanding of physical properties in doped manganites including the magnetic-field-induced IMT, there have been no sound theoretical studies on the electric-fieldinduced IMT phenomenon and a satisfactory theory of this transition is still awaited. Obviously, a profound understanding of this phenomenon not only is fundamental in physics but also has an impact on real applications. In this work, we shall answer two important and key questions in detail: (i) what is the origin of this IMT phenomenon? and (ii) how it occurs in realistic systems? Based on a well-accepted DE Hamiltonian with the long-range Coulomb interaction and the electric field included, we investigate the electric-fieldinduced switching effect in manganites. Using a selfconsistent mean-field approach to handle the effective Hamiltonian that takes a part strong correlation into account, it is found that the electric field can suppress the $\mathrm{CO}$, and even lead to the IMT via changing the magnetic configuration, driving the system from an $\mathrm{AF}$ and $\mathrm{CO}$ state to an $\mathrm{FM}$ and gapless state. Moreover, for realistic manganites with intrinsic inhomogeneities, we make a numerical simulation study on the electric-field-induced IMT. Our results can naturally account for the experimentally observed IMT.

Based on the well-known extended DE Hamiltonian for manganites ${ }^{13}$ and by including the long-range Coulomb interaction as well as the electric field, we may write the total Hamiltonian as

$$
\begin{aligned}
H= & -t \sum_{\langle i j\rangle \sigma}\left(c_{i \sigma}^{\dagger} c_{j \sigma}+\text { H.c. }\right)-J_{H} \sum_{i} \mathbf{S}_{i} \cdot \mathbf{s}_{i}+U \sum_{i} n_{i \uparrow} n_{i \downarrow} \\
& +J_{A F} \sum_{\langle i j\rangle} \mathbf{S}_{i} \cdot \mathbf{S}_{j}+\sum_{i \sigma} \mathbf{F} \cdot \mathbf{r}_{i} n_{i \sigma} \\
& +\sum_{(i j) \sigma \sigma^{\prime}} V_{i j}\left(n_{i \sigma}-n\right)\left(n_{j \sigma^{\prime}}-n\right)
\end{aligned}
$$

where $c_{i \sigma}$ is the electron annihilation operator with spin $\sigma$ and $\mathbf{s}_{i}=\Sigma_{\sigma \sigma^{\prime}} \boldsymbol{\sigma}_{\sigma \sigma^{\prime}} c_{i \sigma}^{\dagger} c_{i \sigma^{\prime}} / 2$ with $\boldsymbol{\sigma}$ as the Pauli matrices, $\mathbf{S}_{i}$ (treated classically, $S=1$ ) is the local spin on each Mn site 

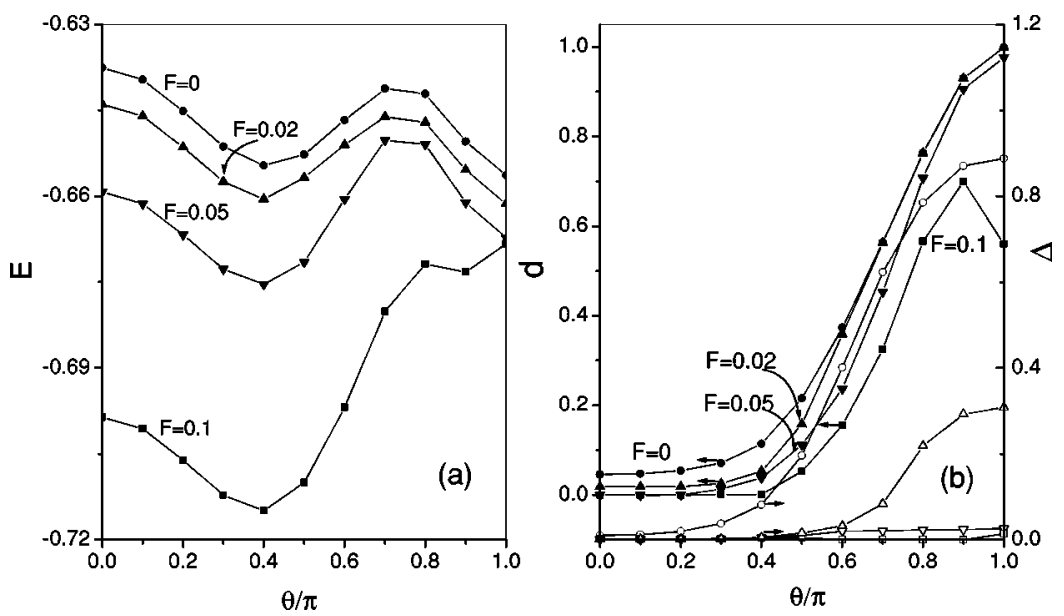

FIG. 1. The calculated (a) energy per site, $E$, and (b) charge-ordering parameter $d$ (block symbols) and energy gap $\Delta$ (open symbols) vs $\theta$. The circles, up triangles, down triangles, and squares correspond to $F=0,0.02,0.05$, and 0.1 , respectively. with $J_{A F}$ as the AF coupling between the two nearestneighboring Mn sites, $\mathbf{r}_{i}$ is the dimensionless position of site $i, n_{i \sigma}=c_{i \sigma}^{\dagger} c_{i \sigma}, V_{i j}=V /\left|\mathbf{r}_{i}-\mathbf{r}_{j}\right|$ is the long-range Coulomb interaction, and $n$ is the average density of electrons. $\mathbf{F}$ $=e \mathbf{E} a$ with $\mathbf{E}$ as the applied electric field and $a$ the lattice constant. Here we use $\langle i j\rangle$ and $(i j)$ to denote the nearest neighboring pairs and arbitrary pairs, respectively. In manganites, the on-site repulsion $U$, the Hund's coupling $J_{H}$, and the electronic hopping $t$ usually satisfy the relation $U \gg J_{H}$ $\gg t$. For simplicity, we take the $U \rightarrow \infty$ limit, in which the double occupancy of electrons at the same site is completely forbidden. Meanwhile, due to the large $J_{H}$, the singly occupied state with the electron spin being antiparallel to the local spin can exist only as a virtual process. In the projection approximation, up to the second-order perturbation an effective Hamiltonian of Eq. (1) can be derived as ${ }^{13,14}$

$$
\begin{aligned}
H_{e f f}= & -t \sum_{\langle i j\rangle}\left[\cos \left(\theta_{i j} / 2\right) d_{i}^{\dagger} d_{j}+\text { H.c. }\right] \\
& -\sum_{\langle i j\rangle} \frac{t^{2} \sin ^{2}\left(\theta_{i j} / 2\right)}{J_{H}}\left(n_{i} h_{j}+n_{j} h_{i}\right)+\sum_{i} \mathbf{F} \cdot \mathbf{r}_{i} n_{i} \\
& +\sum_{(i j)} V_{i j}\left(n_{i}-n\right)\left(n_{j}-n\right)+J_{A F} \sum_{\langle i j\rangle} \cos \theta_{i j},
\end{aligned}
$$

where $d_{i}$ is the electron operator that is fully polarized along the localized spin on the same site. $\theta_{i j}$ is the angle between neighboring spins with the Berry phase in the hopping term being neglected. ${ }^{15} n_{i}=d_{i}^{\dagger} d_{i}$ and $h_{i}=1-n_{i}$ are number operators of the particle and hole, respectively. A mean-field approximation is applied to the above equation by decoupling the interaction $n_{i} n_{j} \rightarrow n_{i}\left\langle n_{j}\right\rangle+\left\langle n_{i}\right\rangle n_{j}-\left\langle n_{i}\right\rangle\left\langle n_{j}\right\rangle$ and replacing the spin part with their average values $\left\langle\cos \left(\theta_{i j} / 2\right)\right\rangle$ $=\cos (\theta / 2)$ and $\left\langle\sin ^{2}\left(\theta_{i j} / 2\right)\right\rangle=\sin ^{2}(\theta / 2)$. Then the Hamiltonian reduces to

$$
\begin{aligned}
H_{e f f}= & -t \cos (\theta / 2) \sum_{\langle i j\rangle}\left(d_{i}^{\dagger} d_{j}+d_{j}^{\dagger} d_{i}\right) \\
& +\sum_{i}\left[V_{i}\left(\theta,\left\{\left\langle n_{j}\right\rangle\right\}\right)+\mathbf{F} \cdot \mathbf{r}_{i}\right] n_{i}+E_{0}\left(\theta,\left\{\left\langle n_{j}\right\rangle\right\}\right),
\end{aligned}
$$

where $V_{i}=\Sigma_{\delta_{i}} t^{2} \sin ^{2}(\theta / 2)\left(1-2\left\langle n_{\delta_{i}}\right\rangle\right) / J_{H}+\sum_{j \neq i} V_{i j}\left\langle n_{j}-n\right\rangle$, with $\delta_{i}$ being the nearest neighbor of site $i$, and $E_{0}$ is the energy constant from mean-field decoupling.

To study the stability of the insulating state, we diagonalize Eq. (3) in an $L \times L$ square lattice at half doping $n=1 / 2$ using the iteration self-consistently. The parameters are taken to be $J_{H}=6, J_{A F}=0.08$, and $V=0.2$, with $t=1$ the unit of energy, and the lattice size $L=30$. By summing the energy of the occupied electronic states together with $E_{0}$, the total energy can be readily obtained and its $\theta$ dependence is plotted in Fig. 1(a). In the absence of the electric field, $F=|\mathbf{F}|=0$, there are two local minima, one at $\theta \approx 0.4 \pi$ and the other at $\theta=\pi$. Their energies are rather close to each other, but the state at $\theta=\pi$ has slightly lower energy, corresponding to a perfect AF configuration. In the presence of an electric field, which is applied along the $x$ direction, we also present the total energy as a function of $\theta$ for $F=0.02,0.05$, and 0.1 . In our calculation, we have chosen the center of the lattice as the origin of coordinates, where the potential due to the electric field is equal to zero. It can easily seen from curves in Fig. 1(a) that the lowest-energy state of the system is shifted to $\theta \approx 0.4 \pi$, at $F=0.05$ and 0.1 which has a large component of the FM configuration. The local minimum at $\theta \approx 0.4 \pi$ implies that once the system is driven to this FM state by the electric field, it may still be quite stable after the electric field is reduced, especially at low temperatures. This feature is consistent with the hysteretic switching effect observed in experiments. $^{7-11}$ We also find that for fixed $\theta$, a twosublattice type $\mathrm{CO}$ shows up from a self-consistent calculation, and the order parameter $d=\sum_{i} n_{i} e^{i \mathbf{Q} \cdot \mathbf{r}_{i}} / N$, with $\mathbf{Q}$ $=(\pi, \pi)$ and $N$ being the number of sites, is shown in Fig. 1(b) by curves with block dots $(F=0)$, block up ( $F$ $=0.02)$, and down $(F=0.05)$ triangles, and block squares $(F=0.1)$. At $F=0$ and 0.02 , the physical order parameter for $\mathrm{CO}$ corresponds to that at $\theta=\pi$ where $d \approx 1$ and the system is a perfect Wigner crystal structure. When a strong electric field ( $F=0.05$ and 0.1$)$ is applied, the physical order parameter is suppressed to almost zero at $\theta \approx 0.4 \pi$. The suppression of the $\mathrm{CO}$ by the electric field is twofold: one is that the electric field leads to a slope of potential energy in real space which drives electrons move towards the direction of lower potential energy, as a feedback, the effective potential 
energy due to electron-electron interaction is altered, which further changes the charge distribution so that the $\mathrm{CO}$ is weakened. More important, in manganites, the degrees of the charge and magnetic configurations are closely coupled to each other. From the second term in Eq. (2), the effective AF superexchange is favored by the charge-ordered configuration, meanwhile the effective repulsion interaction responsible for the $\mathrm{CO}$ is also favored by the $\mathrm{AF}$ configuration, so that the reduction of the $\mathrm{CO}$ due to the electric field weakens the AF interaction, resulting in a change of the magnetic configuration from AF to FM; such a change further suppresses the CO. So here we see interestingly that the electric field can also change the magnetic configuration ${ }^{7,10}$ due to the coupling between the charge and magnetic configurations, just like what the magnetic field does. In Fig. 1(b), the suppression of the $\mathrm{CO}$ is mainly due to such a change of the magnetic configuration. The critical electrical field $F_{c}$ for this $\mathrm{AF}$ and $\mathrm{CO}$ state to an FM state transition depends on the strength of the interaction $V$ and the superexchange $J_{A F}$, and increases with the increase of them. At $V=0.5, F_{c}$ reaches about 0.05 and 0.2 for $J_{A F}=0.05$ and 0.08 , respectively. It is worth mentioning that in the present calculation we have used a minimal model for manganites based on the $\mathrm{DE}$, in which only the long-range Coulomb interaction is included. Both the long-range and short-range interaction attribute to the $\mathrm{CO}$, but in the presence of an external electrical field only the former can prevent the charge accumulation in regions of lower potential energy due to the field, that is the reason why the long-range interaction must be taken into account. Another parameter we are interested in is the energy gap $\Delta$, which is defined as the difference between the energy of lowest unoccupied (LU) and highest occupied (HO) single electronic states. For our purpose of studying the stability of an insulator as well as the occurrence of the IMT when an electric field is applied, we need to examine whether or not there is an energy gap. The energy gap $\Delta$ as a function of $\theta$ is shown in Fig. 1(b) by curves with open circles $(F=0)$, open up $(F=0.02)$ and down $(F=0.05)$ triangles, and open squares $(F=0.1)$. When $F=0$, the energy gap corresponding to the lowest-energy state at $\theta=\pi$ has a large value $\Delta$ $\approx 0.9$. After the application of a strong electric field at $F$ $=0.05$ or $0.1, \Delta$ is dramatically reduced to practically zero at $\theta=0.4 \pi$, indicating the occurrence of the IMT. It is found that this reduction of $\Delta$ is related to two factors: one is the suppression of the $\mathrm{CO}$, the other is the different potential energies of the $\mathrm{HO}$ and LU states due to the electric field. An observation of the wave functions of the $\mathrm{HO}$ and LU states (not shown here) reveals that both states are extended in the whole lattice for small and medium $\theta(\theta<0.7 \pi)$, while for large $\theta$ they are localized on sites with different electric potentials. In the latter case, since the states at the Fermi level are localized, the system is always an insulator.

So far, we have clearly shown that the IMT can be induced by the application of an electric field. Now let us make a rough estimation on the magnitude of the electric field required. From $F=e a|\mathbf{E}| \sim 0.01 t$ and $t \sim 0.1 \mathrm{eV}$, and $a$ $\approx 4 \AA$, we estimate the electric field $|\mathbf{E}| \sim 10^{5} \mathrm{~V} / \mathrm{cm}$. If such a field is exerted uniformly on a manganite sample with typical size of centimeter, the required voltage reaches $10^{5} \mathrm{~V}$, which is about two orders larger than that reported in Ref. 7 and may destroy the sample. To solve this puzzle, the intrinsic inhomogeneity of manganites must be taken into account. In usual samples, the bulk is not a uniform insulator or metallic state, instead, it consists of nanometer-size or micrometer-size clusters, with each cluster being in either an insulator or metallic state. ${ }^{16,17}$ Such an inhomogeneity is believed to be caused by the phase separation (PS), and the mesoscopic scale of the PS domains is due to the long-range Coulomb interaction, which breaks the large clusters into small ones to avoid a macroscopic charge accumulation. ${ }^{13}$ Since it is not the goal of the present work to investigate the mechanism of the formation of the clusters, we here, by assuming their existence, perform a numerical simulation on a square-lattice percolation model, in which a pair of neighboring sites are connected by a resistor. Initially, the resistance of a resistor can be either $R_{L}$ in the lower-resistance (LR) state or $R_{H}\left(\gg R_{L}\right)$ in the higher-resistance (HR) state, with probability $p$ and $1-p$, respectively. The justification of such a resistor-network model has been made in literatures. ${ }^{18}$ Once a voltage is applied to the system, the resistance of a resistor can switch from $R_{H}$ to $R_{L}$ if the exerted bias voltage exceeds the critical value $V_{c}$. For a given initial resistor configuration, we first calculate the bias voltage on each single resistor. If there are simultaneously more than one HR resistors whose bias voltages exceed $V_{c}$, we switch the resistor whose bias voltage is the largest. Then, since the whole voltage distribution is affected by this change, we repeat the above procedure until no HR resistor is under the bias voltage above(or on) $V_{c}$. By using this method, the threshold voltage $V_{t h}$ required to induce the IMT at a different concentration $p$ is calculated and shown in Fig. 2(a). Here and below in this paragraph, IMT refers to the sharp drop of resistance of the system, not necessarily the switch of all HR resistors, and the "Insulator" and "Metal" marked in Fig. 2 are distinguished by this sharp drop; they do not mean that all resistors are in HR and LR states. The simulation is performed in an $M \times M$ lattice for $M=100$ and $120, R_{L}=1$ and $R_{H}=10^{6}$, and averaged over 40 resistor configurations. Near the percolation critical point around $p=0.5$ in the HR side, $V_{t h}$ is close to $V_{c}$, which means that in this case there is a filamentary path connecting two electrodes in which all but one resistors are $R_{L}$. When a voltage is applied between the electrodes, along this path almost the entire voltage drop is exerted on the only one HR resistor so that the critical value $V_{c}$ of this single resistor is also the threshold voltage $V_{t h}$ of the whole system. In general, for an initially fixed resistor configuration, if in a certain path the number of HR resistors is $N_{H}$, then a voltage above $N_{H} V_{c}$ exerted on the two ends of this path is sufficient to switch all these HR resistors, so the upper limit of $V_{t h}$ is $N_{H}^{\min } V_{c}$, where $N_{H}^{\min }$ is the minimum of $N_{H}$ among all possible paths between electrodes. Once the applied voltage exceeds $V_{t h}$, all the $N_{H}^{\min }$ HR resistors in the corresponding path are switched to $R_{L}$ and this path is turned into a metallic one, leading to the IMT. Usually, $N_{H}^{\min }$ is much less than $M$ and it decreases with the increase of $p\left(N_{H}^{\min }\right.$ is zero for $p$ above the percolation critical point), therefore $V_{t h}$ is much lower than $M V_{c}$, with the 

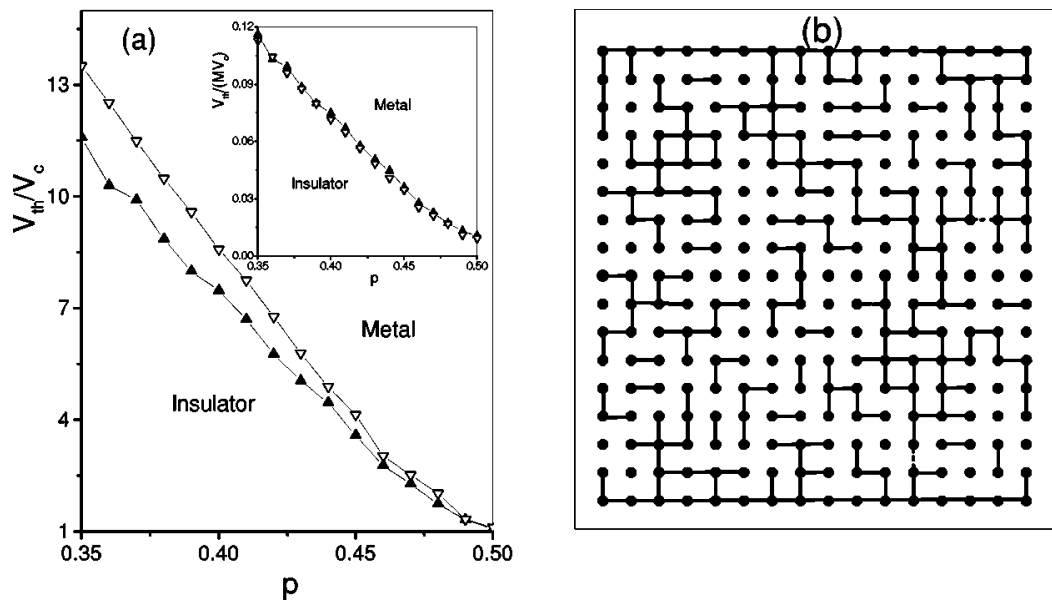

FIG. 2. (a) The threshold voltage to induce the IMT as a function of the concentration in the percolation simulation in the $M \times M$ square lattice for $M=100$ (block symbols) and 120 (open symbols), with average over 40 resistor configurations. In the inset, $V_{t h}$ is scaled to the lattice size. (b) A typical resistor configuration in a 16 $\times 16$ lattice under a bias voltage $2 V_{c}$ between the top and bottom lines, solid lines between neighboring sites represent $R_{L}$ and dashed lines are resistors switched from $R_{H}$ to $R_{L}$ due to the electric field. latter as the threshold voltage required when the electric field is exerted uniformly on the system. A typical resistor configuration for $M=16$ is shown in Fig. 2(b), in which under a bias voltage of $2 V_{c}$, two HR resistors are switched and the IMT has occurred. So for doped manganites, one interesting difference between the application of a magnetic field and that of an electric field is that the former is more uniform while the latter is highly nonuniform due to the inhomogeneity of the sample, which results in a relatively small voltage to induce the IMT. The actual magnitude of $V_{t h}$ depends on both $V_{c}$ and the concentration $p$. $V_{c}$ is related to the size of the cluster: the smaller the size is, the smaller $V_{c}$ is, and from the magnitude of electric field in Fig. 1 it is estimated that for nanometer-size and micrometer-size clusters, $V_{c}$ should be in orders of $0.01 \mathrm{~V}$ and $10 \mathrm{~V}$, respectively. The concentration varies with the change of the compositions in samples. That might be a reason why $V_{t h}$ can be in different orders of magnitude for $\left(\mathrm{La}_{1-y} \mathrm{Pr}_{y}\right)_{0.7} \mathrm{Ca}_{0.3} \mathrm{MnO}_{3}$ at $y=1$
(Ref. 7) and $0.5:^{8}$ the concentration of metallic regions in the latter is expected to be higher as $\mathrm{La}_{0.7} \mathrm{Ca}_{0.3} \mathrm{MnO}_{3}$ possesses metallic properties.

To conclude, we have theoretically studied the electricfield-induced IMT in doped manganites. The transition can be understood in the framework of the DE mechanism, in which the presence of an electric field can suppress the charge ordering and drive the system from the AF state with an energy gap at the Fermi level to the FM and gapless state, triggering the IMT. The intrinsic inhomogeneity in manganites may also play an important role in the observed IMT phenomenon.

The authors thank Professor N.J. Wu and Dr. Yan Chen for valuable discussions. This work was supported by a grant from Texas ARP grant (Grant No. ARP-003652-0241-1999), the Robert A. Welch Foundation, and the Texas Center for Superconductivity at the University of Houston.
${ }^{1}$ A. Urushibara, Y. Moritomo, T. Arima, A. Asamitsu, G. Kido, and Y. Tokura, Phys. Rev. B 51, 14103 (1995); Y. Tomioka, A. Asamitsu, Y. Moritomo, H. Kuwahara, and Y. Tokura, Phys. Rev. Lett. 74, 5108 (1995).

${ }^{2}$ S. Uhlenbruck et al., Phys. Rev. Lett. 82, 185 (1999); Y. Endoh et al., ibid. 82, 4328 (1999).

${ }^{3}$ H. Kuwahara, Y. Tomioka, A. Asamitsu, Y. Moritomo, and Y. Tokura, Science 270, 961 (1995); Y. Tomioka, A. Asamitsu, H. Kuwahara, Y. Morimoto, and Y. Tokura, Phys. Rev. B 53, R1689 (1996).

${ }^{4}$ Y. Morimoto, H. Kuwahara, Y. Tomioka, and Y. Tokura, Phys. Rev. B 55, 7549 (1997).

${ }^{5}$ V. Kiryukhin, D. Casa, J.P. Hill, B. Keimer, A. Vigliante, Y. Tomioka, and Y. Tokura, Nature (London) 386, 813 (1997).

${ }^{6}$ K. Miyano, T. Tanaka, Y. Tomioka, and Y. Tokura, Phys. Rev. Lett. 78, 4257 (1997); M. Fiebig, K. Miyano, Y. Tomioka, and Y. Tokura, Science 280, 1925 (1998).

${ }^{7}$ A. Asamitsu, Y. Tomioka, H. Kuwahara, and Y. Tokura, Nature (London) 388, 50 (1997).

${ }^{8}$ N.A. Babushkina, L.M. Belova, D.I. Khomskii, K.I. Kugel, O.Yu. Gorbenko, and A.R. Kaul, Phys. Rev. B 59, 6994 (1999).

${ }^{9}$ V. Ponnambalam, S. Parashar, A.R. Raju, and C.N.R. Rao, Appl.
Phys. Lett. 74, 206 (1999); C.N.R. Rao, A.R. Raju, V. Ponnambalam, Sachin Parashar, S. Parashar, and N. Kumar, Phys. Rev. B 61, 594 (2000).

${ }^{10}$ J. Sesé, J. García, J. Blasco, and C. Rillo, Phys. Rev. B 61, 11236 (2000).

${ }^{11}$ J. Sakai, A. Kitagawa, and S. Imai, J. Appl. Phys. 90, 1410 (2001).

${ }^{12}$ S.Q. Liu, N.J. Wu and A. Ignatiev, Appl. Phys. Lett. 76, 2749 (2000); A. Beck, J.G. Bednorz, C. Gerber, C. Rossel, and D. Widmer, ibid. 77, 139 (2000).

${ }^{13}$ E. Dagotto, T. Hotta, and A. Moreo, Phys. Rep. 344, 1 (2001).

${ }^{14}$ S.Q. Shen and Z.D. Wang, Phys. Rev. B 59, 14484 (1999); R.Y. Gu, Z.D. Wang, S.Q. Shen, and D.Y. Xing, ibid. 61, 1211 (2000).

${ }^{15}$ M.J. Calderón and L. Brey, Phys. Rev. B 58, 3286 (1998).

${ }^{16}$ M. Uehara, S. Mori, C.H. Chen, and S.-W. Cheong, Nature (London) 399, 560 (1999).

${ }^{17}$ A. Moreo, M. Mayr, A. Feiguin, S. Yunoki, and E. Dagotto, Phys. Rev. Lett. 84, 5568 (2000).

${ }^{18}$ M. Mayr, A. Moreo, J.A. Vergés, J. Arispe, A. Feiguin, and E. Dagotto, Phys. Rev. Lett. 86, 135 (2001); Y. Xiong, S.Q. Shen, and X.C. Xie, Phys. Rev. B 63, 140418 (2001). 